Call for Correspondence JNCCN is committed to providing a forum to enhance collaboration between academic medicine and the community physician. We welcome comments about the NCCN Clinical Practice Guidelines in Oncology (NCCN Guidelines), articles published in the journal, or any other topic relating to cancer prevention, detection, treatment, supportive care, or survivorship. Please submit correspondence to JNCCN.edmgr.com or to JNCCN@nccn.org.

Letters should be no more than 400 words, with no more than 5 references if included. Please include the full names, degrees, and affiliations of all letter authors and a phone number or e-mail address for contact.

Letters are considered for publication as space allows. NCCN reserves the right not to publish correspondence for any reason it deems appropriate. All letters are subject to editing and/or abridgment.

\section{Reply to 'It May be Time to Abandon Urine Tests for Bladder Cancer'}

\author{
"...for whom the bell tolls, It tolls for thee." \\ - John Donne
}

The article, "It May be Time to Abandon Urine Tests for Bladder Cancer"1 reinforces what is widely known within urology: that current urine-based assays for the diagnosis of bladder cancer are subpar. Briefly, the FDA has approved 4 urine-based assays for bladder cancer detection (UroVysion for initial evaluation and tumor surveillance, ImmunoCyt for tumor surveillance, BTA stat for initial evaluation and tumor surveillance, NMP-22 for initial evaluation and tumor surveillance). We have shown that BTA stat is a surrogate for hematuria ${ }^{2}$ and NMP-22 identifies states of cellular proliferation, ${ }^{3}$ and thus it is no wonder one can have positive BTA stat or NMP-22 test results in the face of a benign condition. The lack of robustness is also well documented with ImmunoCyt and UroVysion. ${ }^{4}$ Voided urinary cytology (VUC), the gold standard for noninvasive monitoring, lacks sensitivity, especially in low-grade and low-stage disease. Although VUC is recommended in the American Urological Association (AUA) guidelines for the evaluation of bladder cancer, it is used in $<10 \%$ of these evaluations. ${ }^{5}$ The use of these urine-based assays continues to decline each year. Accordingly, current urine tests for bladder cancer are "dead."

As is evident in most cancers, single biomarkers have inadequate predictive power, and thus the concept that the presence or absence of a single molecular biomarker will aid diagnostic or prognostic evaluation has not proved to be the case. This makes sense when one analyzes the complex interactions between various molecules within a single pathway, the cross-talk between molecular pathways, the redundancy of some pathways, and the oligoclonality of many tumors. Thus, we previously proposed a paradigm shift from single biomarker research to a more global assessment of bladder cancer looking for molecular signatures associated with the disease. Other disease sites have embraced this notion and now have assays in clinical use (eg, Oncotype Dx Colon, MammoPrint, and Genomic Prostate Score).

Although Fantony and Inman ${ }^{1}$ reinforce these points, little information is given about promising new leads. Several groups are on the precipice of potentially bringing such a multiplex assay to the clinic. For example, in a large multicenter study of 789 subjects, Ribal et $\mathrm{al}^{6}$ recently reported that their 5 -gene expression signature consisting of ANXA10, DAB2, HYAL2, SPOCD1, and MAP4K1 possessed a sensitivity and specificity of $81 \%$ and $91 \%$, respectively. At the 2016 AUA meeting in San Diego, in a large cohort of 803 subjects, Lotan et al ${ }^{7}$ reported a 4-gene-expression signature consisting of CDC2, MDK, IGFBP5, and HOXA13, which possessed a sensitivity and negative predictive value of $93 \%$ and $97 \%$, respectively. Furthermore, our group previously reported on a multiplex panel of protein biomarkers consisting of ANG, APOE, A1AT, CA9, IL8, MMP9, MMP10, PAI-1, SDC1, and VEGF.8 After review of pooled data from 999 subjects, meta-analysis showed that the combination of 10 biomarkers possessed a higher log odds ratio (3.61; 95\% CI, 2.90-4.32) than any single biomarker (H.F. and C.J.R., unpublished data; 2017). Subsequently, we launched a multicenter prospective trial to test the assay in patients with hematuria (gross and microscopic, $n=1,600$ ) and those undergoing surveillance for bladder cancer $(n=400)$. Thus, several groups may be within 5 years of having a new validated multiplex assay available for effective cancer risk 
assessment, early detection, and early diagnosis of bladder cancer, ushering in a new era in bladder cancer diagnosis.

Hideki Furuya, PhD, and Charles J. Rosser, MD, MBA

University of Hawaii Cancer Center, Clinical and

Translational Research Program

\section{References}

1. Fantony JJ, Inman BA. It may be time to abandon urine tests for bladder cancer. J Natl Compr Canc Netw 2015;13:1163-1166.

2. Miyake M, Goodison S, Rizwani W, et al. Urinary BTA: indicator of bladder cancer or of hematuria. World J Urol 2012;30:869-873.

3. Miyake M, Goodison S, Giacoia EG, et al. Influencing factors on the NMP-22 urine assay: an experimental model. BMC Urol 2012;12:23.

4. Mbeutcha A, Lucca I, Mathieu R, et al. Current status of urinary biomarkers for detection and surveillance of bladder cancer. Urol Clin North Am 2016;43:47-62.

5. Elias K, Svatek RS, Gupta S, Ho R, Lotan Y. High-risk patients with hematuria are not evaluated according to guideline recommendations. Cancer 2010;116:2954-2959.

6. Ribal MJ, Mengual L, Lozano JJ, et al. Gene expression test for the non-invasive diagnosis of bladder cancer: a prospective, blinded, international and multicenter validation study. Eur J Cancer 2016;54:131-138.

7. Lotan Y, Raman J, Shariat S, et al The development and clinical validation of a high sensitivity urine biomarker test for the determination of recurrence in urothelial carcinoma patients. Presented at American Urological Association 2016 Annual Meeting; May 6-10, 2016; San Diego, CA. Abstract PI-LBA07.

8. Shimizu Y, Furuya H, Bryant Greenwood P, et al. A multiplex immunoassay for the non-invasive detection of bladder cancer. J Transl Med 2016;14:31.

\section{Authors' Reply to Letter to Editor: "Reply to 'It May be Time to Abandon Urine Tests for Bladder Cancer"”}

Furuya and Rosser suggest that the problem with current urine tests is that they are not yet good enough. They argue that with further optimization and development and by combining multiple molecular tests, urine tests for bladder cancer will become dramatically more useful.

Although we agree that urine tests for bladder cancer will probably become more refined and their diagnostic performance (ie, sensitivity and specificity) will improve to a degree, there are more fundamental problems with urine tests for bladder cancer than the tests themselves. In fact, we suggest that the problem lies less with the specific tests and more with the medical decision-making scenario to which they are applied. For example, we recently reported a study of 4,023 cytology-cystoscopy pairs and 1,696 UroVysion fluorescence in situ hybridization (FISH)-cystoscopy pairs that showed very significant and clinically relevant differences in urine test performance by patient characteristics. ${ }^{1}$ Both urine cytology and UroVysion FISH had an increase in sensitivity and a decrease in specificity among older patients, male patients, and former smokers. FISH performed much better in patients with a prior diagnosis of bladder cancer than in those being evaluated for hematuria. ${ }^{1}$ It should be noted that in all of these groups, the performance of these tests was poor. This type of testing problem is known as spectrum bias, and seems to be a particular problem for urine-based bladder cancer tests. It illustrates that great consideration is required regarding how the tests perform in different patient populations, because this appears to substantially affect test performance.

When dealing with a test for cancer, false-positive and false-negative results can lead to serious consequences, and in the case of urine tests for bladder cancer, this actually happens quite frequently. Test manufacturers often claim that a positive urine 
test is "accurate" even when the gold standard cystoscopy is negative. They claim that this false-positive test will eventually become a true-positive if you follow the patient over time with more cystoscopies, a phenomenon that has been termed the anticipatory positive test. That more cystoscopies will eventually detect more bladder cancer is, of course, guaranteed, because bladder cancer tends to recur over time due to the biology of the disease, and regardless of the urine test result. The issue of false-positive versus anticipatory positive urine tests was carefully examined for FISH and cytology in a recent publication. ${ }^{2}$ The take-home message is that although a positive urine test is associated with an increased probability of developing a future bladder cancer, it is not a guarantee of a future cancer. Many of these initially false-positive urine tests remain falsely positive, even when credited up to a year of follow-up time in which the tumor they are purportedly detecting is allowed to occur.

One of the main goals of an office-based urine test should be to replace the need for more invasive screening/surveillance, such as a cystoscopy. The penchant for bladder cancer to recur necessitates frequent procedural follow-up, which is costly and has psychosocial ramifications for patients. ${ }^{3}$ To date, no urine test has been developed that has come close to reaching the goal of substituting for cystoscopy, which then begs the question: why are we spending precious healthcare money on urine tests that don't change how bladder cancer surveillance occurs?

Should a combination urine assay be developed that uses several biomarkers, each of which has their own sensitivity/specificity for diagnosing bladder cancer, its overall accuracy will still be subject to the influence of spectrum bias in the population being tested. To illustrate this point, we will use the following scenario: a patient with a history of low-grade, noninvasive bladder cancer is in the office for routine follow-up 1 year after his transurethral resection of bladder tumor. A new urine test that uses 2 biomarkers is going to be used, and the results will dictate whether he needs an office cystoscopy. Biomarker A has excellent sensitivity for detecting recurrent bladder cancer but marginal specificity, whereas biomarker B has marginal sensitivity but excellent specificity for detecting recurrent bladder cancer. This test should prove useful in that biomarker $\mathrm{A}$ would act as a screening test, whereas biomarker B would be confirmatory. However, the uncertainty that is introduced by patient-related factors (eg, demographics, disease state, exposure history) and tumor-related factors creates a scenario in which the interpretation of a positive or negative result could be difficult. A Bayesian approach could be used to interpret the results of this combined assay, and the overall test performance will be affected by how each individual biomarker performs in that particular patient's clinical context.

This scenario is demonstrated in a multicenter study of 2 novel biomarkers being added to urine cytology and FISH in an attempt to improve their clinical performance. ${ }^{4}$ Therefore, given the uncertainty of urine testing, most clinicians would likely just go ahead with office cystoscopy.

Brant A. Inman, MD, MS, and Joseph J. Fantony, MD Duke Cancer Institute, Durham, North Carolina

\section{References}

1. Gopalakrishna A, Longo TA, Fantony JJ, et al. The diagnostic accuracy of urine-based tests for bladder cancer varies greatly by patient. BMC Urol 2016;16:30.

2. Gopalakrishna A, Fantony JJ, Longo TA, et al. Anticipatory positive urine tests for bladder cancer [published online ahead of print January 10, 2017]. Ann Surg Oncol, doi: 10.1245/s10434-016-5763-5.

3. van der Aa MN, Steyerberg EW, Sen EF, et al. Patients' perceived burden of cystoscopic and urinary surveillance of bladder cancer: a randomized comparison. BJU Int 2008;101:1106-1110.

4. Fantony JJ, Longo TA, Gopalakrishna A, et al. Urinary NID2 and TWIST1 methylation to augment conventional urine cytology for the detection of bladder cancer [published online ahead of print January 13, 2017]. Cancer Biomark, doi: 10.3233/CBM-160261. 